\title{
Fréchet's metric spaces-100th next
}

\author{
Milan R. TASKOvić
}

\begin{abstract}
In this survey I give fundamental historical facts on the metric spaces and some further consequences in history of mathematics. In the second part of this survey I give a historical oversee the fundamental facts on transversal spaces as a nature extension of Fréchet's, Kurepa's and Menger's spaces.
\end{abstract}

\section{History}

While the generalization of geometry beyond the Euclidean spaces $\mathbf{R}^{n}$ originated with Italian geometers such as Ascoli, Arzelà, and Volterra, it first received a coherent and abstract treatment in Fréchet's doctoral thesis of 1906.

As Fréchet took steps to delineate functional analysis, and thereby to distinguish it from the calculus of variations, he isolated two important types of generalized spaces: those, which he called $L$-spaces, where the notion of limit was based on an axiomatization of convergent sequences and, among the $L$-spaces, those on which a distance function ${ }^{1}$ could be defined.

Maurice Fréchet in 1905 and in particular in his doctoral dissertation in 1906 considered a distance functional $d: X \times X \rightarrow \mathbf{R}$ between any two given general objects $x, y$ of a given set $X$ such that satisfy four postulates for all $x, y, z$ in the set $X: d(x, y) \geq 0, d(x, y)=0$ if and only if $x=y, d(x, y)=d(y, x)$, and

$$
d(x, y) \leq d(x, z)+d(z, y) .
$$

Hausdorff gave the latter the name of metric spaces in 1914 when he pondered the role of point-sets within abstract set theory. Preferring to investigate metric spaces rather than $L$-spaces, Hausdorff offered an alternative generalization for the

\footnotetext{
${ }^{1}$ Distance function. The notion of distance $d(x, y)$ between points $x, y$ is very old and connected with measurements. Certainly, the notion is present in works of Th a les (Milet, circa 624 B.C. -546 B.C.), one of the seven wise men of the Antic Greece, the first man who predicted the eclipse of the sun (for the year 585 B.C.); he performed various calculations with distances and angles.

A perpetual monument to the old notion of distance is the Pythagora Theorem on triangles with an angle of $90^{\circ}$. The determination of the gravity center of various figures like: triangle, arc, part of a circle, sphere, part of ball,... is a further nice application of the distance, researches tied with the names of: Archimedes, Euclid, Jordanus Nemorarius, Fermat, Guldin, Toricelli, Cavalieri, Descartes, Roberval, Pascal, Ceva, Newton, Leibniz, Clairaut, Ascoli, Arzelà, and Volterra.
} 
former: topological spaces, based on the concept of neighborhood. In effect, he was introducing what are now termed Hausdorff spaces, those topological spaces in which any two distinct points $p$ and $q$ belong to some disjoint open sets $A_{p}$ and $A_{q}$.

As was known, Fréchet relied heavily but implicitly on the Denumerable Axiom to obtain theorems about convergence in $L$-spaces. Hausdorff, on the other hand applied the Axiom of Choice consciously and liberally in his topological theorems, but he rarely remarked where he used it. In 1927, borrowing a term from Fréchet, Hausdorff defined a topological space to be separable if it had a countable dense subset, and then obtained the following result by means of the Denumerable Axiom: Every subspace of a separable metric space is separable.

More generally, he used the Axiom to demonstrate that every metric space has a dense subset of least power. ${ }^{2}$

The fundamental notion tied with a distance function on $X$ is the one on the open ball: given any $x_{0} \in X$ and any real $r>0$ the ball (or spheroid) with a center $x_{0}$ and radius $r$ is defined by

$$
K\left(x_{0}, r\right):=\left\{x \in X: d\left(x, x_{0}\right)<r\right\} ;
$$

and, these notions allow us to associate to any subset $A$ of $X$ the corresponding closure or the adherence $C l A$.

A special feature in the preceding notion is the "triangular relation" in the form (Rt) occurring in the elementary geometry and in many other cases. ${ }^{3}$

Fréchet remarked that this spaces have similar properties. In 1910 he asked whether this two classes of spaces should be the same. E. W. Chittenden in 1917 confirmed this conjecture; a simple proof was exibited by A. H. Frink in 1937.

The possibility of defining such notions as limit and continuity in an arbitrary set is an idea which undoubtedly was first put forward by Fréchet in 1905, and developed by him in his famous thesis in 1906.

The simplest and most fruitful method which he proposed for such definitions was the introduction of the notion of distance. But the greatest merit of Fréchet lies in the emphasis he put of three notions which were to play a fundamental part in all later developments of Functional Analysis ${ }^{4}$ : compactness, completeness, and separability.

\footnotetext{
${ }^{2}$ From Hausdorff in 1927: A subset $M$ of a topological space $X$ is dense if every point of $X$ is either a member of $M$ or a limit point of $M$.

${ }^{3}$ Fréchet's further step. At the same time, Fréchet considered instead of (Rt) the following, apparently weaker, regularity condition: There exists a selfmapping $f$ of $\mathbf{R}_{+}:=(0,+\infty)$ into itself such that $f(x) \rightarrow 0(x \rightarrow 0)$ and that $d(a, b)<x, d(b, c)<x$ implies that $d(a, c)<f(x)$ for all $a, b, c \in X$.

${ }^{4}$ Shortly after 1920 there arose among Luzin's students at Moscow a new school of topologists. Foremost among them were Paul Alexandroff and Paul Urysohn, whose researches focused on compactness and metrization. In contrast to $\mathrm{L} u \mathrm{z}$ in whose sympathies remained constructivist, Alexandroff and Urysohn were strongly influenced by Hausdorff's ideas and employed the Axiom of Choice quite freely. Alexandroff and Urysohn, generalized Fréchet's version of compactness, originally called bicompactness.
} 
In the same year that Čech's article appeared, two generalizations of convergencedirected sets and filters-were developed independently of each other in the United States and France. A few years later, these two generalizations would provide a way to represent compactness as well. In 1922 the first approach had been formulated jointly by two mathematicians, E. H. Moore of the University of Chicago and H. L. Smith of the University of the Philippines.

Birkhoff considered the three fundamental topological notions to be convergence, closure, and neighborhood. Through revising the generalized limit introduced by Moore and Smith, Birkhoff obtained such a notion in which denumerability was no longer essential. ${ }^{5}$

Meanwhile Henry Cartan proposed to the Paris Academy of Sciences a different but related generalization of convergence: filters. Cartan formulated and proved a fundamental result later known as the Ultrafilter Theorem: Every filter can be extended to an ultrafilter.

In 1940 Cartan's characterization of compactness enabled Bourbaki to give a proof of Tychonoff's Theorem ${ }^{6}$ restricted to Hausdorff's spaces, by using ultrafilters rather than complete accumulation points: The product of any family of compact Hausdorff spaces is a compact Hausdorff space.

At Princeton, Tukey in 1940 lauded the theory of directed sets as more intuitive than that of filters, since it remained closer to sequential convergence.

\footnotetext{
${ }^{5}$ In the same vein, one of Hadamard's doctoral students, Maurice Fréchet, had relied heavily on set theory in his thesis of 1906. There he generalized analysis and developed what he called the functional calculus. Influenced heavily by Arzelà, Fréchet laid the foundations for an abstract treatment of functional analysis.

Fréchet used limits of sequences to approach problems which we now consider topological, and so he required the Denumerable Axiom.

Like Borel when introducing Borel sets and like Lebesgue when proposing his Measure Problem, Fréchet broadened the analytic concept of limit by axiomatizing it via his notion of $L$-class.

Also, he next established the well-known generalization of a topological theorem of Cantor's. The proof, which Fréchet described as typical of those in his thesis, relied on denumerably many arbitrary choices to pick an element from each of given sets.

Indeed, the Denumerable Axiom aided Fréchet in many of his results. One important example concerned his concept of extremal set and second when Fréchet extended Arzelà's Theorem to extremal sets.

On the other hand, he presented to the Paris Academy of Sciences the first results from his thesis, namely his axiomatization of limits, on 21 November 1904. This was presumably before he was aware of Zermelo's Axiom of Choice.

${ }^{6}$ Yet the result for which he is best known, later named Tychonoff's Theorem, did not occur there in its general form: The product of any family of compact spaces is compact.

Rather, Tychonoff in 1930 proved only that the product of any number of copies of the closed interval $[0,1]$ is compact.

One topologist who undertook such a generalization was the Czechoslovakian Eduard Cech, then at Brno. In 1937 Cech was apparently the first to recognize that Tychonoff's Theorem lay hidden in the latter's paper of 1930 and to demonstrate it in full generality. For, in contrast to the Moscow topologists, Cech did not restrict himself to Hausdorff spaces but stated his results for arbitrary topological spaces.
} 
In the preceding spaces, the progress of the mankind is visible spectaculary in the extension of cases and situations in which, given two distinct objects $A$ and $B$, a distance $d(A, B)$ from $A$ to $B$ is determinable. ${ }^{7}$ In this sense we have the following new facts in name as transversal spaces.

Let $X$ be a nonempty set. The function $\rho: X \times X \rightarrow \mathbf{R}_{+}^{0}:=[0, \infty)$ is called an upper transverse on $X$ (or upper transversal) if: $\rho[x, y]=\rho[y, x], \rho[x, y]=0$ iff $x=y$, and if there is a function $g:\left(\mathbf{R}_{+}^{0}\right)^{2} \rightarrow \mathbf{R}_{+}^{0}$ such that

$$
\rho[x, y] \leq \max \{\rho[x, z], \rho[z, y], g(\rho[x, z], \rho[z, y])\}
$$

for all $x, y, z \in X$. An upper transversal space is a set $X$ together with a given upper transverse on $X$. The function $g:\left(\mathbf{R}_{+}^{0}\right)^{2} \rightarrow \mathbf{R}_{+}^{0}$ in $(\mathrm{A})$ is called upper bisection function. ${ }^{8}$

A fundamental first example of upper transversal space for the upper bisection function $g:\left(\mathbf{R}_{+}^{0}\right)^{2} \rightarrow \mathbf{R}_{+}^{0}$ defined by $g(a, b):=a+b$ is a metric space. Also, if the upper bisection function $g:\left(\mathbf{R}_{+}^{0}\right)^{2} \rightarrow \mathbf{R}_{+}^{0}$ defined by $g(a, b):=\max \{a, b\}$, then we obtain ultrametric space.

On the other hand, the function $\rho: X \times X \rightarrow \mathbf{R}_{+}^{0} \cup\{+\infty\}:=[0, \infty]$ is called a lower transverse on $X$ (or lower transversal) if: $\rho[x, y]=\rho[y, x], \rho[x, y]=+\infty$ iff $x=y$, and if there is a lower bisection function $d:[0, \infty]^{2} \rightarrow[0, \infty]$ such that

$$
\min \{\rho[x, z], \rho[z, y], g(\rho[x, z], \rho[z, y])\} \leq \rho[x, y]
$$

for all $x, y, z \in X$. A lower transversal space is a set $X$ together with a given lower transverse on $X$.

We remarked that an important example of upper transversal spaces is also and every Fréchet's space with the regularity condition. For this an upper bisection function $g$ : $\left(\mathbf{R}_{+}^{0}\right)^{2} \rightarrow \mathbf{R}_{+}^{0}$ can be defined by $g(\alpha, \beta):=\max \{x, f(x)\}$.

Further, as a natural extension of transversal (upper and lower) spaces we have the following notations of space.

\footnotetext{
${ }^{7}$ In this respect it is very instructive to be aware of some typical situations like distance between the Earth and the Moon, the problem of geodesics on a given surface or in a given manifold (an example as Einstein's theory of relativity). During the past 20th Century the terrestrian human beings extended tremendously their capacity to determine $d(A, B)$ for bodies $A, B$ belonging to the microscopic as well as to the megascopic world very far in the Universe and outside on the Solar and Galactic system.

${ }^{8}$ In 1926 Lind en baum [Fund. Math, 8 (1926), p. 111.] has shown that condition $d(x, y)=$ $d(y, x)$ of the Fréchet's axioms may be omitted from the postulate for a metric space if condition (Rt) is replaced by

$$
d(x, y) \leq d(x, z)+d(y, z)
$$

for all $x, y, z \in X$. For this fact see a paper of Frink in 1937. Also see book of Tasković in 2001.

On the other hand, similarly to Fréchet, Frink in 1937 instead relation (Rt) to avail one's self of the following facts: first, if $d(x, y)<\varepsilon$ and $d(z, y)<\varepsilon$, then $d(x, z)<2 \varepsilon$-generalized triangle property; and, second: for every $\varepsilon>0$ there exists $\xi(\varepsilon)>0$ such that if $d(x, y)<\xi(\varepsilon)$ and $d(z, y)<\xi(\varepsilon)$, then $d(x, z)<\varepsilon$-uniformly regular. Second condition reduces to first condition if $\xi(\varepsilon)=\varepsilon / 2$.
} 
Let $X$ be nonempty set and let $P:=(P, \preceq)$ be a partially ordered set. The function $\rho: X \times X \rightarrow P$ is called an upper ordered transverse on $X$ (or upper ordered transversal) if: $\rho[x, y]=\rho[y, x]$, and if there is an upper bisection function $g: P \times P \rightarrow P$ such that

$$
\rho[x, y] \preceq \sup \{\rho[x, z], \rho[z, y], g(\rho[x, z], \rho[z, y])\}
$$

for all $x, y, z \in X$. An upper ordered transversal space is a set $X$ together with a given upper ordered transverse on $X$.

Let $k=\aleph_{\alpha}(\alpha \geq 0)$ be a regular cardinal. Call a topological space $X$ an upper $k$-transversal space or a $g\left(D_{\alpha}\right)$-space if there exists $\rho: X \times X \rightarrow \omega_{\alpha} \cup\left\{\omega_{\alpha}\right\}:=$ $W$ such that: $\rho[x, y]=\omega_{\alpha}$ iff $x=y, \rho[x, y]=\rho[y, x]$, and if there is $g: W^{2} \rightarrow W$ such that $(\mathrm{Bs})$ for all $x, y, z \in X$.

Obviously, Fréchet's ordered spaces are important examples of upper $k$-transversal spaces.

Open problem 1. Does for every regular cardinal $k \geq \aleph_{0}$ there exists an upper $k$-transversal nonlinearly orderable topological space?

In connection with the preceding, the function $\rho: X \times X \rightarrow(P, \preceq)$ is called a lower ordered transverse on $X$ (or lower ordered transversal) if: $\rho[x, y]=$ $\rho[y, x]$ and if there is a lower bisection function $d: P \times P \rightarrow P$ such that

$$
\inf \{\rho[x, z], \rho[z, y], d(\rho[x, z], \rho[z, y])\} \preceq \rho[x, y]
$$

for all $x, y, z \in X$. A lower ordered transversal space is a set $X$ together with given lower ordered transverse on $X$.

On the other hand, let $k=\aleph_{\alpha}(\alpha \geq 0)$ be a regular cardinal. Call a topological space $X$ a lower $k$-transversal space or $d\left(D_{\alpha}\right)$-space if there exists the function $\rho: X \times X \rightarrow \omega_{\alpha} \cup\left\{\omega_{\alpha}\right\}:=W$ such that: $\rho[x, y]=\omega_{\alpha}$ iff $x=y, \rho[x, y]=\rho[y, x]$ and if there is $d: W^{2} \rightarrow W$ such that (Bi) for all $x, y, z \in X$.

Open problem 2. Does for every regular cardinal $k \geq \aleph_{0}$ there exists a lower $k$-transversal nonlinearly orderable topological space?

We notice, in connection with this problem, that work of Đ. K urepa in 1963 is very important, where there is result that for every regular cardinal $k \geq \aleph_{\alpha}(\alpha \geq 0)$ there exists a $k$-metrizable nonlinearly orderable topological space. A proof of this result was exhibit by S. Todorčević in 1981.

Let $k \geq \aleph_{\alpha}(\alpha \geq 0)$ be a regular cardinal. Call a topological space $X$ a $k$-metrizable space or a $D_{\alpha}$-space if there exist $\rho: X \times X \rightarrow \omega_{\alpha} \cup\left\{\omega_{\alpha}\right\}$ and $\phi: \omega_{\alpha} \rightarrow \omega_{\alpha}$ such that: $\rho(x, y)=\omega_{\alpha}$ iff $x=y, \rho(x, y)=\rho(y, x), \rho(x, y)>\phi(\varepsilon)$ and $\rho(y, z)>\phi(\varepsilon)$ implies $\rho(x, z)>\varepsilon$, and that the sets

$$
B_{\varepsilon}(x)=\{y \in X: \rho(x, y)>\varepsilon\}
$$

for $x \in X$ and $\varepsilon<\omega_{\alpha}$ are form a basis of $X$.

This definition was given by Đ. Kurepa in 1934 using the name pseudo-distancial spaces. The class of all $D_{0}$-spaces is just the class of all metrizable spaces. The class of all pseudo-distancial spaces was extensively considered by Đ. Kurepa, Fréchet, Doss, Colmez, Appert, Papić, Mamuzić, Cammaroto, Kočinac, Ky Fan and many others. 
This class has also the name "spaces with linearly ordered basis of uniformity". We notice, that, an important example of lower ordered transversal spaces is and every Kurepa's pseudo-distancial space. For this a lower bisection function $d: P \times P \rightarrow P$ can be defined by $d(a, b):=\inf \{\varepsilon, \phi(\varepsilon)\}$.

Obviously, Kurepa's pseudo-distancial spaces are important examples of lower $k$-transversal spaces.

On the other hand, let $\tau=\omega_{\mu}$ be a regular cardinal number, $X$ a set and $(G,+, \preceq)$ a linearly ordered abelian group with cofinality $\operatorname{cof}(G)=\omega_{\mu}$ at the identity element $0 \in G$ (which means that 0 is the infimum of a strictly decreasing $\tau$-sequence $\left\{x_{\alpha}: \alpha \in \tau\right\} \subset$ $G \backslash\{0\})$. An $\tau$-metric on $X$ is a function $\rho: X \times X \rightarrow(G, \preceq)$ which satisfies all the formal properties of metric.

This definition of space $X$ was given by Đ. Kurepa in 1934 an by Sikorski in 1950 using the name $\omega_{\mu}$-metrizable topological space (if its topology can be induced by some $\omega_{\mu}$-metric on $\left.X\right)$.

Obviously, $\omega_{\mu}$-metrizable topological spaces are fundamental examples of upper transversal ordered spaces with the upper bisection function $g: G^{2} \rightarrow G$ defined by $g(a, b):=$ $a+b$.

Open problem 3. Find sufficient and necessary conditions such that an upper or a lower $k$-transversal space is the form of a Kurepa's space (a metric space or uniform space or a Fréchet's space with the regularity condition)!?

In the theory of metric spaces, as and in the transversal spaces, it is extremely convenient to use a geometrical language inspired by classical geometry.

Thus elements of a transversal space will usually be called points. Given an upper transversal space $(X, \rho)$, with the upper bisection function $g: P \times P \rightarrow P$ and a point $a \in X$, the open ball of a center $a$ and radius $r \in P$ is the set

$$
g(B(a, r))=\{x \in X: \rho[a, x] \prec r\},
$$

till for given a lower transversal space $(X, \rho)$, with the lower bisection function $d: P \times P \rightarrow P$ and a point $a \in X$, the open ball of center $a$ and radius $r \in P$ is the set

$$
d(B(a, r))=\{x \in X: r \prec \rho[a, x]\} .
$$

We notice that K. Menger introduced in 1928 and 1942 the notion of probabilistic metric space. As an important example of lower ordered transversal spaces we have a Menger's (probabilistic) space.

For the further facts on transversal spaces see papers of Tasković in 1998 and further. Also, the new book of Tasković to refer to transversal spaces and further their applications.

\section{REFERENCES}

[1905] M. Fréchet, La notion d'écart et le calcul fonctionnel, C. R. Acad. Sci. Paris, 140(1905), $772-774$.

[1906] M. Fréchet, Sur quelques points du calcul fonctionnel, Thèse, Paris, 1905. Rendiconti Circolo Mat. Palermo, 22(1906), 1-74.

[1928] K. Menger, Untersuchungen über allgemeine Metrik, Math. Ann., 100(1928), 75-163.

[1934] Đ. R. Kurepa, Tableaux ramifiés d'ensambles. Espaces pseudo-distanciés, C. R. Acad. Paris, 198(1934), 1563-1565. 
[1935] Đ. R. Kurepa, Ensambles ordonnés et ramifiés, Thèse, Paris, 1935, p.p. 1-138; Publ. Inst. Math. Belgrade, 4(1935), 1-138.

[1998] M. R. Tasković, Transversal spaces, Math. Moravica, 2(1998), 133-142.

[2001] M. R. Tasković, Nonlienar Functional Analysis, Second Book: Monographs - Global Convex Analysis - General convexity, Variational methods and Optimization, Zavod za udžbenike i nastavna sredstva i Vojnoizdavački zavod, Beograd 2001, (in Serbian), 1223 pages.

[2003] M. R. Tasković, Transversal intervally spaces, Math. Moravica, 7(2003), 87-102.

[2003a] M. R. Tasković, Survey on transversal normed spaces, Math. Moravica, 7(2003), 149-170.

[2003b] M. R. Tasković, Fixed points on transversal edges spaces, Math. Moravica, 7(2003), $171-182$.

[2004] M. R. Tasković, Theory of transversal point, spaces, and forks, Monographs of a new mathematical theory, Beograd 2004, 1000 pages, (In Serbian), to appear.

Mathematical Faculty

11000 Belgrade, P.O. Box 550

Serbia \& Montenegro

E-mail address: tassneza@eunet.yu 